\title{
Two Novel Defensin Genes from Brassica Juncea and Camelina Sativa Confers Antifungal Activity Against Pathogenic Fungi Alernaria brassicae
}

\author{
GDG Chaturani ${ }^{1 *}$, Zahoor Ahmad Mir ${ }^{2}$ and Anita Grover ${ }^{3}$ \\ ${ }^{1}$ PhD Scholar, National Institute of Plant Biotechnology, New Delhi, India \\ ${ }^{2}$ Senior Research Fellow, National Institute of Plant Biotechnology, New Delhi, \\ India \\ ${ }^{3}$ Principle Scientist, National Institute of Plant Biotechnology, New Delhi, India \\ *Corresponding Author: GDG Chaturani, PhD Scholar, National Institute of Plant \\ Biotechnology, New Delhi, India.
}

Received: November 02, 2021

Published: January 28, 2022

(C) All rights are reserved by GDG Chaturani., et al.

\begin{abstract}
Alternaria blight, incited by A. brassicae is one of the most common and destructive fungal pathogens in Indian mustard which accounts for $46 \%$ of yield loss. Efforts are being made in order to have sufficient knowledge about the genes induced during infection and their regulation measures. We hear report two novel defensins, BjDef and CsDef which confers antifungal activity against $A$. brassicae. During the search of defensins, cloning and sequencing resulted in two open reading frames of 234 bp with characteristic $\alpha$-helix, triple strand antiparallel $\beta$-sheets, highly conserved eight cysteines and an identical backbone structure stabilized by four intra-molecular disulphide bridges in their peptide chains. Amplified fragments were cloned into pET29a vector system and were expressed in Escherichia coli. Recombinant defensin proteins were optimally expressed in isopropyl thio-galactoside concentration of $1.0 \mathrm{mM} / \mathrm{ml}$ and $6 \mathrm{~h}$ of incubation. Purified proteins with hexa-histidine tag by Ni-NTA column exhibited a $16 \mathrm{kDa}$ single fragment. The purified proteins showed an inhibitory effect on hyphal growth indicating disrupted mycelial cells while percentage mycelial growth inhibition calculations indicate inhibition effect of proteins in a concentration dependent manner. Even though at lower concentration inhibition was high by BjDef, at higher concentrations CsDef conferred comparatively more effective inhibition. Relative expression of $C s D e f$ revealed a high level of expression at $48 \mathrm{~h}$ in response to Alternaria infection. Comparatively low level of expression was reported by BjDef. Our results confirmed the ability of above defensins to be used in construction of transgenic brassica to confirm resistance to $A$. brassicae.
\end{abstract}

Keywords: Alternaria Blight; Brassica Juncea; Defensin; Antifungal

\section{Introduction}

Alternaria blight of cruciferous caused by A. brassicae, is one of the most common and destructive disease of Indian mustard (Brassica juncea) and the yield losses have been estimated to range from 35 to $46 \%$ [23]. Infection on silique adversely affects normal seed development, seed weight, seed colour; percent oil content and overall seed quality [22]. Due to severe losses caused by the disease, several attempts have been made to find the sources and defense mechanisms against Alternaria blight. Thus far, methods of disease prevention and control are based only on combining agricultural management practices with chemical control. Conventional breeding to develop resistant cultivars in $B$. juncea against $A$. brassicae is confounded due to non-availability of suitable resistant sources within the available germplasm of cultivated species of Brassica [25], though some varieties differ in their resistance level. Comparatively, broccoli and cauliflower exhibited moderate resistance [26] whereas a high degree of resistance has been found in wild relatives of Brassica outside the tribe Brassicaceae including Camelina sativa [15]. Biotechnological approaches can successfully

Citation: GDG Chaturani., et al. "Two Novel Defensin Genes from Brassica Juncea and Camelina Sativa Confers Antifungal Activity Against Pathogenic Fungi Alernaria brassicae". Acta Scientific Agriculture 6.2 (2022): 40-49. 
be utilized to develop resistant variety of $B$. juncea provided that molecular mechanism of defense is delineated. Efforts are being made in order to have sufficient knowledge about the genes induced during infection and their regulation measures.

During their life cycle plants are infected by various pathogens including bacteria, virus and fungi. Appropriate regulation of defense responses is important for plant fitness, as activation of defense responses has deleterious effects on plant growth [12]. The molecular communication between plant and pathogens starts almost immediately after the pathogen makes contact with the plant surface [3], and plants liberate different chemicals which interfere with activities of the pathogen and pathogenesis, thereby preventing or reducing infection [8]. During pathogen invasion, production and accumulation of pathogenesis related proteins (PR) have been reported in many plant species suggestive of a general role for these proteins in adaptation to biotic stress conditions [19]. Among these, a group of small cysteine-rich proteins known as defensins [33] classified as the PR-12 family [31], displays antimicrobial activities against micrographic fungi [39]. Defensins are described to interact with the negatively charged molecules present at the cell membrane of pathogens, causing an increase of its permiabilization, leading to cell leakage and death by necrosis [16]. They act by inhibiting pre-existing ion channels or form new membrane pores that disrupt cellular ion balance and thereby interfering with pathogen nutrition retarding their development, thus contributing to plant disease resistance $[6,8,13]$. One of the plant defensin genes from $B$. juncea has been isolated and its similarity with gamma thionin and knottin families of plant antimicrobial peptides reported [29]. According to the studies, expression of BjDefensin gene increases significantly upon Alternaria infection, JA and wounding, but not by SA. Furthermore, they reported the pathogen-inducible expression of BjDefensin promoter after fungal infection.

Defensin proteins with clearly demonstrated antifungal activities has led to their deployment for enhancing disease resistance in crop plants and reported to have been effective in several cases [24] including B. napus and was effective against the blackleg fungus Leptospheiri maculans [37]. Tobacco and peanut plants constitutively expressing the mustard defensin has conferred resistant to F. moniliform and C. arachidicola [2], whereas B. napus with defensin gene Ovd from Orychophragmus violaceus against S. sclerotiorum also showed resistance [38]. Banana plants overexpressing Petunia floral defensins, PhDef1 and PhDef2 against infection of $F$. oxysporum was effective [11] and expression of barley antifungal defensin genes from heterologous sources in $B$. juncea against $A$. brassicae has also been reported [7]. There are very few reports of antifungal activity of defensin proteins from $B$. juncea against $A$. brassicae. In this paper we report cloning and sequencing of two novel defensin genes from B. juncea and C. sativa, expression of these genes in $E$-coli system and their antifungal activity against A. brassicae.

\section{Materials and Methods}

Cloning and sequencing of defensin genes from $B$. juncea and C. sativa

Available defensin genes (CDS sequences) of $B$. juncea and related species were downloaded from NCBI database. According to the consensus sequences of these CDS sequences, primers were designed by Primer3Plus software http://www.bioinformatics.nl/ cgi-bin/primer3plus/primer3plus.cgi, such that full length of the CDS of defensin genes could be recovered from B. juncea and $C$. sativa cDNA. Total RNA was isolated from leaf tissues of $B$. juncea and C. sativa using TRIzol reagent (Invitrogen ${ }^{\mathrm{TM}}$ ) and first strand cDNA synthesis was performed from $2 \mu \mathrm{g}$ of total RNA using Superscript III cDNA synthesis kit (Invitrogen ${ }^{\mathrm{TM}}$ ) following the manufacturer's instructions. PCR was performed to amplify the defensin genes from isolated cDNA. PCR conditions included an initial denaturing step at $94{ }^{\circ} \mathrm{C}$ for $4 \mathrm{~min}$ followed by 30 cycles of $94{ }^{\circ} \mathrm{C}$ for $30 \mathrm{~s}, 55$ ${ }^{\circ} \mathrm{C}$ for $30 \mathrm{~s} 72{ }^{\circ} \mathrm{C}$ for $1 \mathrm{~min}$. PCR products were analyzed by electrophoresis on $1.7 \%$ agarose gel and fragments of expected size were eluted out from the gel using QIAquick ${ }^{\circledR}$ Gel Extraction Kit according to the manufacturer's instruction. Isolated fragments were cloned into pGEM-T easy vector by TA cloning kit; pGEM ${ }^{\circledR}$-T Easy Vector System 1 (Promega) and transformed into DH5 $\alpha$. Colonies with confirmed transfromants were sequenced by Sanger sequencing and sequences were deposited in NCBI Gene Bank database.

\section{Characterization of defensin genes}

By using the ExPASy translate tool (https://web.expasy.org/ translate/), coding sequences were translated to peptide sequences. Conserved domains of all non-redundant sequences obtained were analyzed by the NCBI-CDD database (https://www.ncbi.nlm. nih.gov/Structure/cdd/wrpsb.cgi) and SMART (http://smart.embl-heidelberg.de/). To confirm that identified protein sequences were members of defensin family, Pfam (https://pfam.xfam.org/) analysis was performed. Three dimensional secondary structures 
of isolated proteins were constructed from ProMod3 Version 1.3.0. homology modeling method by SWISS-MODEL server (https:// swissmodel.expasy.org/interactive). Computer analysis of the amino acid sequence to compute chemical and physical parameters were performed with ProtParam tool on the ExPASy server (https://web.expasy.org/protparam/) and possible disulphide bridges were determined from the DISULFIND server (http://disulfind.dsi.unifi.it/). The subcellular localization of each defensin protein of $B$. juncea and $C$. sativa was predicted by Plant-mPLoc (http://www.csbio.sjtu.edu.cn/bioinf/plant-multi/). The defensin sequences obtained by sequencing were employed to identify respective genes from $B$. juncea and $C$. sativa genome from a local blast in BioEdit V 7.0.4. software. The exon-intron structure of $B$. juncea defensin encoding genes were determined based on alignment of coding sequences with corresponding genome sequences and graphical display was created by online Gene Structure Display Server (http://gsds.cbi.pku.edu.cn/). Conserved cis-acting regulatory elements in the promoter region of the putative defensin genes were analyzed by plant care database (http://bioinformatics.psb. ugent.be/webtools/plantcare/html/). Evolutionary relationships among isolated defensins and already available B. juncea, B. rapa, B. oleracea B. napus and A. thaliana defensins, were analyzed by neighbor-joining phylogenetic method using MEGA 7.0 software based on the amino acid sequences of the defensin proteins.

\section{Cloning of defensin genes in expression vector}

Primers were designed with ECoRI and HinDIII restriction sites with termination codons removed. Genes were amplified by PCR from Def-pGEM-T construct. PCR products were separated by electrophoresis on $1.7 \%$ agarose gel and gel elution was performed to isolate the genes using QIAquick ${ }^{\circledR}$ Gel Extraction Kit according to the manufacturer's instruction. Restriction digestion was done with ECoRI and HinDIII restriction enzymes both in the isolated genes and pET29a vector (Novagen) separately and then ligated. Ligated products were transformed into BL21 by heat shock method and transformation was confirmed by colony PCR. Plasmid isolation was performed using NucleoSpin ${ }^{\circledR}$ Plasmid isolation kit following manufacturer's instruction from the confirmed transfromants and plasmid PCR was performed for further confirmation. All PCR conditions included an initial denaturing step at $94{ }^{\circ} \mathrm{C}$ for 4 min followed by 30 cycles of $94{ }^{\circ} \mathrm{C}$ for $30 \mathrm{~s}, 55^{\circ} \mathrm{C}$ for $30 \mathrm{~s} 72{ }^{\circ} \mathrm{C}$ for $1 \mathrm{~min}$.

Protein induction by IPTG, purification and quantification

Protein expression was induced by incubating with $0,0.5,1.0$, 1.5, $2.0 \mathrm{mM}$ IPTG at $37^{\circ} \mathrm{C}$ for $3,6,9,12 \mathrm{~h}$. Cultures were then pel- leted down by centrifugation at 11,000 rpm for $1 \mathrm{~min}$ and dissolved in $200 \mu \mathrm{l}$ of lysis buffer. Sonication was carried out with 3 cycles 30 $\mathrm{s}$ of each. The sonicated product was centrifuged at 11,000 rpm for $1 \mathrm{~min}$, supernatant was separated and pellet was re-suspended in $200 \mu \mathrm{l}$ of lysis buffer separately. To confirm the protein induction $15 \mu \mathrm{l}$ of protein sample was mixed with $6 \mu \mathrm{l}$ of Lamelli sample buffer, incubated at $100{ }^{\circ} \mathrm{C}$ for $10 \mathrm{~min}$, kept on ice for $5 \mathrm{~min}$ and then were loaded on to SDS-PAGE gel along with pre stained protein marker and electrophoresed at room temperature. At the end of electroporation, gel was stained with Coomasssie stain for $2 \mathrm{~h}$, and de-stained in a de-staining solution for $4 \mathrm{~h}$ with concomitant renewal of the solution. Protein purification was carried out using QIAexpress ${ }^{\circledR}$ Ni-NTA Fast Start protein purification kit. Total protein in each sample was estimated by Bradford method using BSA as standard.

Evaluation of antifungal activity of BjDef and CsDef against A. brassicae

To evaluate the antifungal activity of defensin proteins, radial diffusion assay [5] was performed for crude proteins as well as purified proteins. A. Brassicae was grown on potato dextrose agar (PDA) medium and when mycelial colony expanded, holes were made $5 \mathrm{~mm}$ away from the rim of the mycelium and different concentrations of purified protein was added. Lysis buffer was used as the control. Cultures were then incubated at $25{ }^{\circ} \mathrm{C}$ till they grow past the control. To quantify the antifungal activity of defensin proteins, radial diffusion assay was performed individually for different concentrations of purified proteins. Fungal plugs were placed on PDA medium containing different concentrations of purified protein with lysis buffer as the control and incubated at $26^{\circ} \mathrm{C}$. When colony expanded, radial growth of the mycelium was measured and percentage of mycelial growth inhibition was measured using following formula assay [5].

Mycelial growth in control - Mycelial growth in protein x 100

Mycelial growth in control

Expression analysis of BjDef and CsDef in response to A. brassicae infection

$B$. juncea and $C$. sativa plants with six leaves, were treated with conidial suspensions of $A$. brassicae isolated from disease infected plants. Sporulated mycelia from 21-day old cultures were used to prepare the conidial suspensions by suspending in sterilized distilled water and adjusted to $5 \times 10^{3}$ conidia/ml by a hemacytometer. 
Total RNA was isolated from leaf samples collected at different time intervals using TRIzol reagent (Invitrogen ${ }^{\mathrm{TM}}$ ). First strand cDNA synthesis was performed using Superscript III cDNA synthesis kit (Invitrogen ${ }^{\mathrm{TM}}$ ) following the manufacturer's instructions. Primers were designed using Primer Quest $\AA$ online primer design software (https://eu.idtdna.com/pages/tools/primerquest). Quantitative real time PCR was performed using SYBR Premix Ex Taq Kit (Takara ${ }^{\mathrm{TM}}$ ) with the reaction conditions as $95^{\circ} \mathrm{C}$ for $10 \mathrm{~min}, 40$ cycles of $95{ }^{\circ} \mathrm{C}$ for $10 \mathrm{~s}$ and $55^{\circ} \mathrm{C}$ for $30 \mathrm{~s}$. $\beta$-tubulin was used as the reference gene for relative expression analysis and relative quantification method $(\Delta \Delta \mathrm{CT})$ was used to evaluate quantitative variation.

\section{Results and Discussion}

Cloning, sequencing and Characterization of $B$. juncea and $C$. sativa defensins

Sequencing of the cloned cDNA resulted in two defensin ORFs each from B. juncea and C. sativa (NCBI Acc. No: BjDef-MN369575 and CsDef-MN369576) of 243 bp length. Computer analysis of physical and chemical parameters of defensin proteins revealed that both the peptides are 78 aa in length and their molecular weight 8.69 and $8.64 \mathrm{kDa}$ respectively. The theoretical isoelectric points were 9.91 and 10.75 while instability index values were
44.05 and 32.75 respectively. A protein whose instability index is smaller than 40 is predicted as stable and a value above 40 predicts that the protein may be unstable. Instability index indicates that BjDef was stable while CsDef was unstable. Instability index provides an estimate of the stability of the protein in a test tube. For cell survival and development, stability of the cellular proteins is a fundamental requirement. Positive values (0.037and 0.186 respectively) of grand average hydropathy (GRAVY) defines above proteins as polar. Proteins with positive GRAVY score are hydrophobic in nature and mostly reside in membranes while negative are hydrophilic and globular proteins. The subcellular localization predicted that the above proteins were resided in vacuole consistent with the GRAVY value.

Multiple sequence alignment showed the highly conserved eight cysteine which is the signature of plant defensins and share an identical backbone structure stabilized by four intramolecular disulphide bridges (Figure 1). This observation is consistent with the finding of previous records [17] which suggested that the conservation of these residues may be due to their roles in providing stability and in the folding mechanism, especially the cysteines involved in the formation of disulfide bridges.

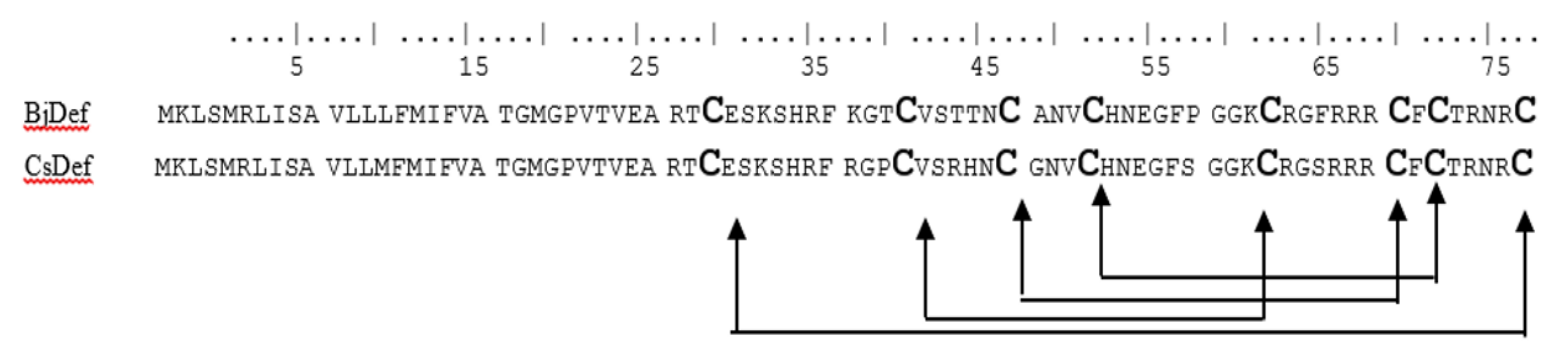

Figure 1: Amino acid alignment of BjDef and CsDef showing conserved eight cysteines and four disulphide bridges.

The three-dimensional structure predictions determined that both proteins are characterized by $\alpha$-helix and triple strand antiparallel $\beta$-sheets (Figure 2). The $\alpha$-helix is connected to the second $\beta$-sheet through a cysteine stabilized $\alpha / \beta$ motif. Despite their similarity in structure, the amino acid sequence variation between the two defensins shows $10 \%$ dissimilarity. This variation between primary sequences may account for different biological roles attributed to plant defensins which include antibacterial activity [1] and antifungal activity.

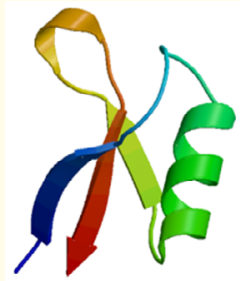

BjDef

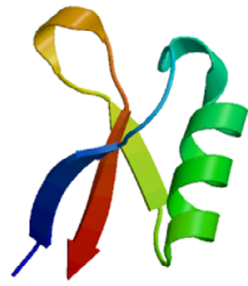

CsDef
Figure 2: Three-dimensional structure of BjDef and CsDef with $\alpha$-helix and triple strand antiparallel $\beta$-sheets. 
Specific sequence diversity and structural feature homology are characteristic features of defensin proteins. Plant defensins were first identified from wheat and barley grains [32] and were initially called gamma-thionin because of their similarity to thionins in terms of size and cysteine content. Therefore, those proteins containing gamma-thionin domain under Knot1 superfamily were defined to be belonging to the defensin family. The gamma-thionin domain under Knot1 superfamily of CsDef and BjDef were identified and similar protein structure and domain compositions were found (Figure 3), demonstrating that the protein structure is remarkably conserved within the plant defensins. It has also been reported that the plant defensins are structurally similar to their insect counterparts despite the low amino acid sequence similarity between these two organisms [9] and the presence of Knot1 domain and gamma-thionin domain in their studies on CDef1 in Capsicum. The Knot1 domain or knottins represent plant antimicrobial peptides, plant amylase inhibitors, plant gamma-thionins and arthropod defensins [20].

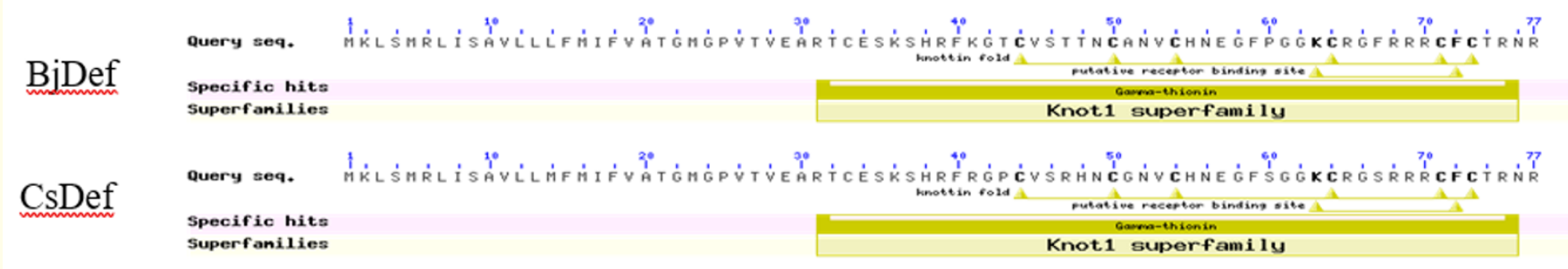

Figure 3: Conserved gamma-thionin domain of knotin superfamily in BjDef and CsDef proteins.

To gain further insight into the structural evolution of BjDef and CsDef, the exon-intron organization was analyzed by comparing the corresponding genomic sequences with their coding sequences through Gene Structure Display software. Based on the predicted schematic structures, both the genes displayed a single intron and two exons (Figure 4). It has been reported that transcription and post transcriptional modification rates determine the overall rate of transcript [14]. The number and length of exon and intron have been reported as important regulatory players in gene expression. New scientific evidence described cells having rapid cell cycles contain gene-architecture towards short genes with few introns, allowing efficient expression during short cell cycles and in genes that need rapid regulation during stress. Therefore, the conserved trend toward shorter gene with fewer introns in BjDef and CsDef may indicate their rapid induction following pathogen attack.

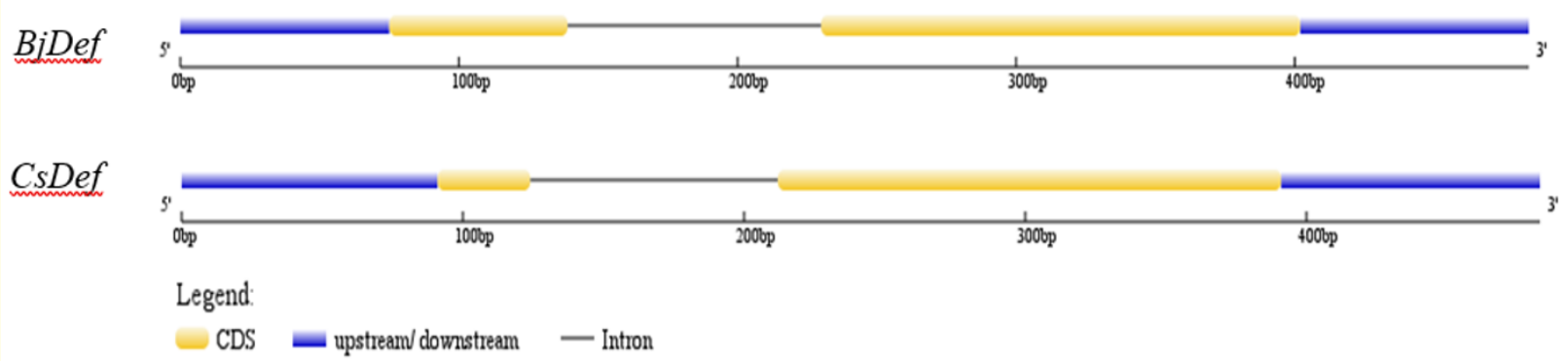

Figure 4: Gene structure of BjDef and CsDef showing a single intron in the coding region.

Regulation of gene transcription is achieved by binding the transcription factors to cis-acting regulatory elements. Some cis-regu- latory elements are involved in stress responses. To analyze how the expression level of BjDef and CsDef respond to stress stimuli, 
$2 \mathrm{~kb}$ upstream promoter regions were scanned for stress-related cis-regulatory elements using the plant CARE online service. Four cis-acting regulatory elements involved in MeJA responsiveness, two from each of CGTA-motif and TGACG-motif were identified in the promoter region of both BjDef and CsDef genes denoting to the possible regulation of above defensin genes under JA responsive pathway. Two light responsive elements GTI and a G-box, TGAelement in auxin responsiveness were identified indicating that these defensin genes might be involved in the response to various light stress and hormone treatments via participating in different regulatory mechanisms. Apart from that, 10 common cis-acting elements in promoter and enhancer region, and 14 core promoter elements around -30 of transcription start site were identified. Presence of cis-acting regulatory elements for drought and anaerobic conditions in the promoter region of CsDef indicate that members of defensin gene family might be able to respond to different abi- otic stresses. Similar pattern for stress responsiveness was not observed in cis-acting regulatory elements in BjDef other than regulatory elements responsible for light responsiveness.

To study the evolutionary relationship of BjDef and CsDef to other related species comprehensively, defensin proteins of $B$. napus, B. oleracea, and B. rapa, B. nigra were obtained from Brassica database and a phylogenetic tree was constructed (Figure 5). According to the analysis all the identified defensins from Brassica species together with Arabidopsis were divided into six distinct groups. Interestingly both BjDef and CsDef fall under the same group indicating a close relationship between the two genes. It was observed that this group has one or few proteins from each species used in the analysis. This indicates that the genes responsible including BjDef and CsDef may have a recent divergence.

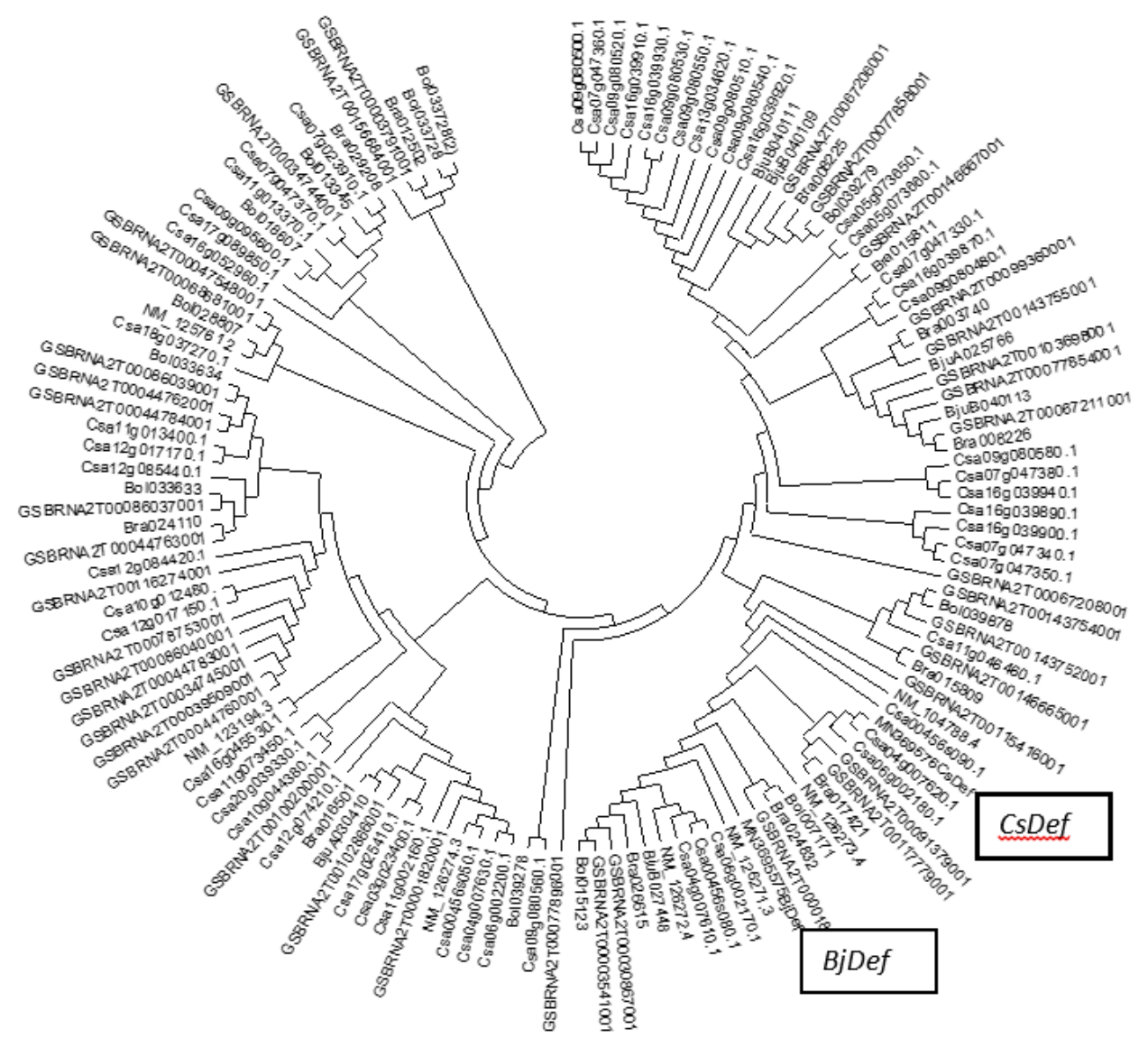

Figure 5: Phylogenetic analysis showing evolutionary relationship of BjDef and CsDef, to B. rapa, B. oleracea, B. napus, C. sativa and A. thaliana defensin proteins by Neighbor-Joining method. 
Cloning and expression of defensins in E. coli system

To clone the above two defensin coding sequences in expression vector, primers were designed with ECoRI and HinDIII restriction sites with termination codon removed to facilitate the expression of hexa-histidine tag. Both defensins were amplified by PCR from Def-pGEM-T construct, subjected to restriction digestion with ECoRI and HinDIII and cloned into pET29a expression vector, which produces a fusion product with histidine tag as a part of short tail sequence of the C-terminus of protein for purification. There are different strategies developed so far to induce the expression of proteins in E-coli. Use of IPTG is the most efficient and effective method to induce promoter expression [4]. Different concentrations of IPTG and incubation periods were tested to induce BjDef and CsDef proteins. Without IPTG, all the extracts of E-coli gave negative results. The expression was significantly influenced by the IPTG concentration and incubation period. A high level of expression of the recombinant protein was obtained after incubation for $6 \mathrm{~h}$ with $1.0 \mathrm{mM}$ IPTG in soluble fraction. Same induction of SsPR10 protein with IPTG has also been reported [18].

Proteins produced in bacteria have three destinations: cytoplasm, periplasm, or secretion into the external medium. However, many E-coli strains are incompetent in translocating produced proteins over their outer membrane. Therefore, highest yields are possible by cytoplasmic expression [30]. CsDef and BjDef production was observed in soluble fraction in the purification process. Expressed proteins purified and analyzed in SDS-PAGE confirmed the presence of both the BjDef and CsDef proteins as single bands. Literature contains various reports of defensins that have been heterologously expressed in divers hosts, such as bacteria, yeast, fungi and plants, mainly for research purposes.The heterologous expression of eukaryotic antimicrobial peptides by microorganisms could be a cost effective tool for functional and clinical research. Furthermore, they show rapid growth on inexpensive substrates, controlled laboratory conditions, and the generally well characterized genetic backgrounds and availability of a wide range of vectors and host strains. Bacterial systems for heterologous protein expression are currently widely used, offering advantages over other methods for protein production, especially for small antimicrobial peptides as well as due to the possibility of isolating and purifying secreted proteins [10]. Among bacteria, E coli is most commonly used for defensin expression [21]. Successful production of recombinant AvBD6, a novel avian $\beta$-defensin by expressing the gene in $E$. coli has also been reported [28]. While the literature reports several cases of successful expression in E. coli, there are also cases where plant defensin production in this vector yielded inactive protein aggregates, which needed to be denatured and refolded [27]. Literature reports the difficulty in obtaining a correctly folded peptide using E. coli for expressed defensin that accumulates in inclusion bodies, so that denaturing extraction and purification are required [21]. A. halleri foliar defensin gene AhPDF1.1 was cloned into and expressed in E. coli with the use of an appropriate oxidative protocol leads to efficient production. The recombinant protein was reported to be active against filamentous fungus Fusarium oxysporum with minimal inhibitory concentration of $0.6 \mu \mathrm{mol} / \mathrm{l}$. Maarof., et al. in 2011 reported CDef1, a cDNA clone that encodes a defensin gene, was isolated from a cDNA library of ripening capsicum annum via differential screening. CDef1 was expressed using the prokaryotic $E$. coli expression system with a $47 \mathrm{kDa}$ fusion peptide.

Evaluation of antifungal activity of defensin proteins against A. brassicae

By using the radial diffusion assay antimicrobial activity of both defensins were clearly demonstrated by the protein's ability to inhibit the growth of $A$. brassicae. The purified proteins of BjDef and $C s D e f$ showed an inhibitory effect on hyphal growth of $A$. brassicae indicating that mycelial cells were disrupted (Figure 6). The inhibitory effect was more by $C s D e f$ compared to BjDef. The hyphal growth was decreased by increasing the concentration of purified protein.

Percentage of mycelial growth inhibition was different between the defensins and the inhibition effects of proteins were in a concentration dependent manner (Figure 7). Although at $4 \mu \mathrm{g} / \mathrm{l}$ concentrations inhibition was high by BjDef, at increased concentrations inhibition was higher by $C s D e f$ indicating a more effective inhibition compared to BjDef. When fungal hyphae were interacted with a plant defensin, the physiological reaction at the plasma membrane leads to a membrane permeability causing $\mathrm{K}^{+}$efflux and $\mathrm{Ca}^{2+}$ influx [34]. Reports indicate that apart from cell permeabilization, plant defensins can also penetrate fungal cell wall and interact with intracellular targets. A pure folded form of AhPDF1.1 of A. halleri demonstrating a strong antifungal activity against $F$. oxysporum has also been reported [21]. $\mathrm{NaD1}$, a novel floral defensin from $\mathrm{Ni}$ cotiana alata showing an antifungal activity against $F$ oxysporum by creating pores at the plasma membrane, entering into the cell and inducing the production of reactive oxygen species and subsequent cell death [36]. 


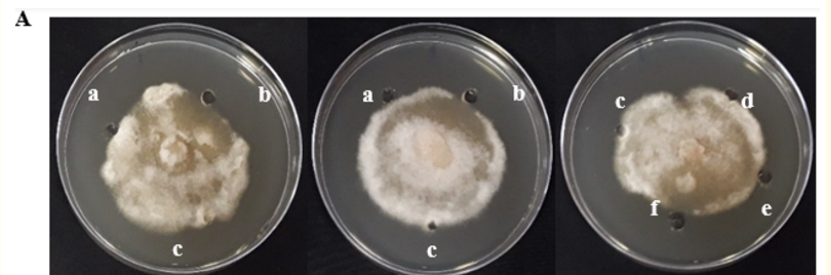

B

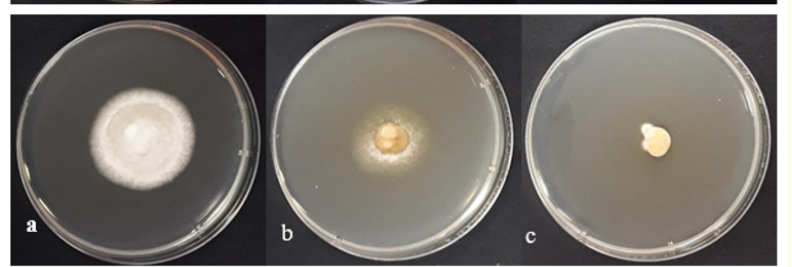

Figure 6: A: Mycelial growth inhibition of A. brassicae by $C s D e f$ and BjDef a: crude protein, b: purified protein, c: control, d: 2

$\mu \mathrm{g} / \mathrm{l}$, e: $5 \mu \mathrm{g} / \mathrm{l}, \mathrm{f}: 10 \mu \mathrm{g} / \mathrm{l}$. B: Mycelial growth inhibition of

A. brassicae a: control b: BjDef and c: CsDef with the concentration of $10 \mu \mathrm{g} / \mathrm{l}$.

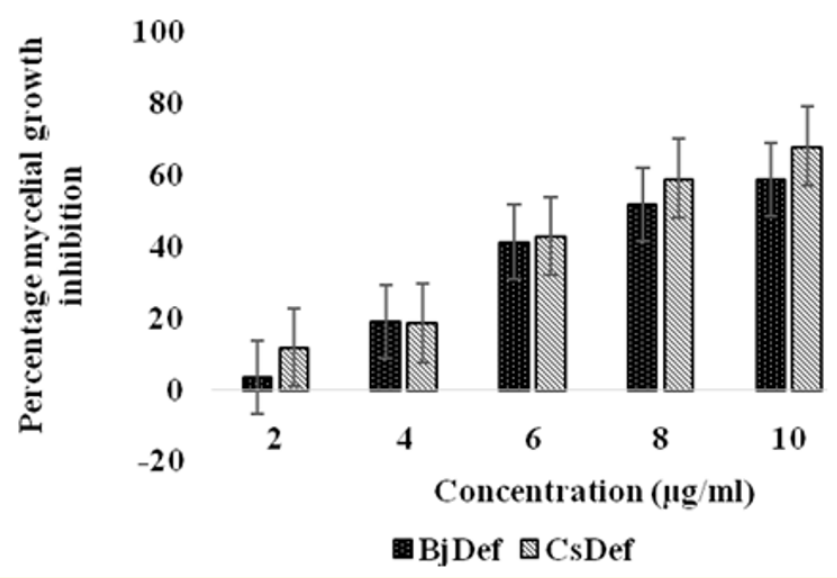

Figure 7: Percentage mycelial growth inhibition of $A$. brassicae by BjDef and CsDef.

Expression analysis of BjDef and CsDef in response to A. brassicae infection

To analyze the expression pattern of BjDef and $C s D e f$ in response to $A$. brassicae infection, plants were inoculated with a spore suspension of $A$. brassicae, and cDNA from leaf samples were analyzed at different time intervals (Figure $8 \mathrm{~A}$ and $8 \mathrm{~B}$ ). Quantitative real time PCR analysis indicated that, relative expression of $C s D e f$ in response to Alternaria infection is comparatively high. However in $C$. sativa, CsDef showed a high induction starting from $12 \mathrm{~h}$ after inoculation and reaching to a peak at $24 \mathrm{~h}$ and then a gradual decrease
(Figure 9). It has been suggested that since necrotrophic pathogens benefited from host cell death upon infection a set of plant defense responses were activated by JA and Ethylene signaling. A. brassicae belongs to the category of necrotrophic fungi and presence of JA responsive elements in promoter regions leads to the conclusion that expression of BjDef and CsDef upon A. brassicae infection is through JA signaling pathway. It is suggested that expression of defensin genes in susceptible species may be visible in latter stages of disease progression or in low concentrations that is not sufficient to arrest the lesion development [35]. There are reports on the induced expression of plant defensin PDF1.2 in B. juncea upon the infection of $A$. brassicae. In locally challenged leaves of $B$. juncea with A. brassicae, a slight induction of PDF1.2 has been observed as early as $2 \mathrm{~h}$ of post inoculation [25].

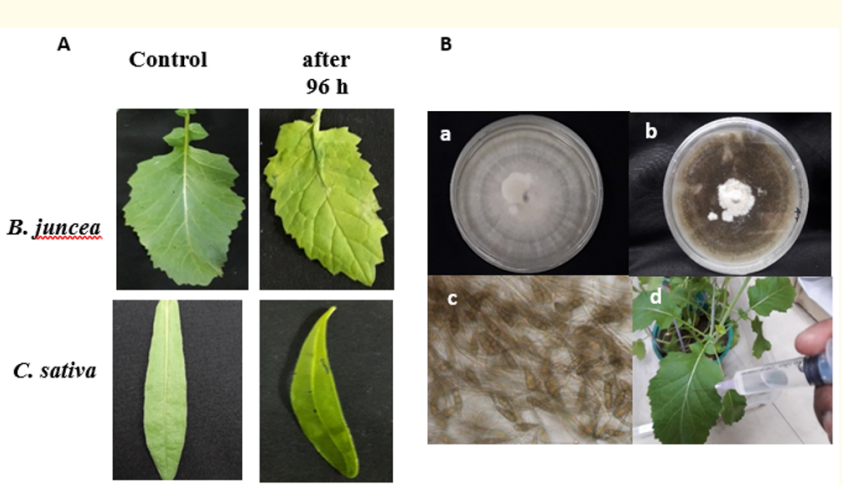

Figure 8: A: $B$. juncea and $C$. sativa leaves before and after infection by $A$. brssicae, B a: Fungal culture of $A$. brassica in PDA medium, b: sporulation culture of $A$. brassicae in RRA medium, c: spore suspension of $A$. brassicae, d: inoculating plant leaves with spore suspension of $A$. brassicae using a hypodermic needle.

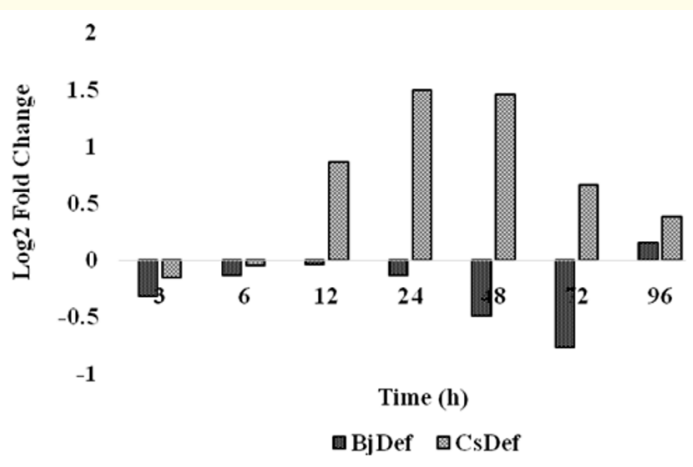

Figure 9: Real-time PCR analysis of defensin genes in B. juncea and $C$. sativa in response to Alternaria infection. 


\section{Conclusion}

Both CsDef and BjDef peptides have the characteristic properties of plant defensins which contain gamma-thionin domain under Knot1 superfamily. Both the proteins are characterized by $\alpha$-helix and triple strand antiparallel $\beta$-sheets in their three-dimensional structure and highly conserved eight cysteines in their amino acid sequence. Expression of these two novel genes CsDef and BjDef are induced by fungal pathogen $A$. brassicae indicating their role in plant defense. The purified proteins clearly demonstrated the antifungal activity against $A$. brassicae during in-vitro assay. Both purified proteins have inhibitory effect; at higher concentrations inhibition was higher by $C s D e f$. In addition to being antimicrobial, plant defensins are also involved in abiotic stress response, as well as plant growth and development. All the results suggest a possible use of these two defensin genes in construction of transgenic mustard against $A$. brassicae which is a problematic fungal pathogen in mustard cultivation in India.

\section{Acknowledgments}

Authors wish to acknowledge the support given by National Phytotron Facility, and fellowship funding from Indian Council for Agricultural Research, India.

\section{Bibliography}

1. Aerts AM., et al. "The mode of antifungal action of plant, insect and human defensins". Cell Molecular and Life Sciences 65 (2008): 2069-2079.

2. Anuradha TS., et al. "Transgenic tobacco and peanut plants expressing a mustard defensin show resistance to fungal pathogens". Plant Cell Reports 27 (2008): 1777-1786.

3. Aoun M. "Host defense mechanisms during fungal pathogenesis and how these are overcome in susceptible plants". International Journal of Botany 13.2 (2017): 82-102.

4. Brian L., et al. "A self-inducible heterologous protein expression system in Escherichia coli". Scientific Reports 6 (2016): 33037.

5. Broguel KE., et al. "Transgenic plants with enhanced resistance to the fungal pathogen Rhizoctonia solani". Science 254 (1991): 1194-1197.

6. Buonaurio R. "Infection and plant defense responses during plant bacterial infection". In: Barka, E. and Clement, C. (eds.) (2008)". Plant Microbe Interactions (2008): 169-197.
7. Chhikara S., et al. "Combined expression of a barley class II chitinase and type I ribosome inactivating protein in transgenic Brassica juncea provides protection against Alternaria brassicae". Plant Cell and Organ Culture 108.1 (2011): 83-89.

8. Doughari JH. "An overview of plant immunity". Journal of Plant Pathology and Microbiology 6 (2015): 322-333.

9. Gachomo EW., et al. "Structural characterization of plant defensing protein superfamily". Molecular Biology Reports 39.4 (2012): 4461-4469.

10. Gaiser RA., et al. "Production of eukaryotic antimicrobial peptides by bacterial". (In) Vilas, M. (ed). Science against microbial pathogens. Formatex Research Center 3 (2011): 992-1002.

11. Ghag SB., et al. "Petunia floral defensins with unique prodomains as novel candidates for development of Fusarium wilt resistance in transgenic banana plants". Plos One 7 (2012): e39557.

12. Glazebrook J. "Contrasting mechanisms of defense against biotrophic and necrotrophic pathogens". Annual Review in Phytopthology 43 (2005): 205-227.

13. Guest D and Brown J. "Plant defense against pathogens". Australian vice chnacellors' committee Canberra (1980): 263-285.

14. Heyn P., et al. "Introns and gene expression: Cellular constraints, transcriptional regulation, and evolutionary consequences". Bioassays 37 (2015): 148-154.

15. Kumar D., et al. "Alternaria blight of oilseed Brassicas: A comprehensive review". African Journal of Microbiology Reports 8.30 (2014): 2816-2829.

16. Lacerda AF., et al. "Antifungal defensins and their role in plant defense". Frontiers in Microbiology 5.116 (2014): 1-10.

17. Lay FT., et al. "Isolation and properties of floral defensin from ornamental tobacco and petunia". Plant Physiology 131 (2003): 1283-1293.

18. Liu YJ., et al. "Solution structure of the plant defensin VrD1 from mung bean and its possible role in insecticidal activity against bruchids". Protein. Structure and Functional Bioinformatics 63 (2006): 777-786.

19. Loon LCV and Strien EAV. "The families of pathogenesis related proteins, their activities, and comparative analysis of PR-1 type proteins". Physiology and Molecular Plant Pathology 55 (1999): 85-97. 
20. Maarof E., et al. "Cloning and heterogeneous expression of CDef1, a ripening induced defensin in Capsicum annum". Australian Journal of Crop Science 5.3 (2011): 123-134.

21. Marques L., et al. "Production of an Aabidopsishalleri foliar defensins in Escherichia coli". Journal of Applied Microbiology 106 (2009): 1640-1648.

22. Meena PD., et al. "Alternaria blight: a chronic disease in rapeseed mustard". Journal of Oilseed Brassica 1.1 (2010): 1-11.

23. Mishra A., et al. "Molecular cloning and in silico analysis of functional homologous of hypersensitive response genes induced during pathogenesis of Alternaria Blight in two genotypes of Brassica". Journal of Protein and Bio Informatics 3 (2010): 244-248.

24. Moosa A., et al. "Transgenic expression of antifungal pathogenesis-related proteins against phyto-pathogenic fungi- 15 years of success". Israel Journal of Plant Sciences 6 (2017): 122-134.

25. Nanayakkara NMC., et al. "Differential expression of defenserelated genes in Synapsis alba and Brassica juncea upon the infection of Alternaria brassicae". Tropical Agriculture Research 27.3 (2016): 123-136.

26. Nowicki M., et al. "Alternaria black spot of Crucifers: Symptoms, importance of disease, and perspectives of resistance breeding". Transgenic Research 19 (2012): 373-384.

27. Padovan L., et al. "Techniques for plant defensin production". Current Protein and Peptide Science 11.3 (2010): 231-239.

28. Peng KS., et al. "Tissue distribution, expression, and antimicrobial activity of Anus platyrphynchos avian $\beta$-defensin 6". Plant Science 92.1 (2013): 97-104.

29. Rawat S., et al. "Isolation and expression analysis of defensin gene and its promoter from Brassica juncea". Journal of Plant Diseases and Protection 124.6 (2017): 591-600.

30. Sahdev S., et al. "Production of active eukaryotic proteins through bacterial expression system: a review of the existing biotechnology strategies". Molecular Cell Biochemistry 307 (2008): 249-264.

31. Sels J., et al. "Plant Pathogenesis-related (PR) proteins: A focus on PR peptides". Plant Physiology and Biochemistry 46 (2008): 941-950.

32. Stotz HU., et al. "Plant defensins: defense, development and application". Plant Signal Behave 4 (2009): 1010-1012.
33. Terras FR., et al. "Small cysteine-rich antifungal proteins from radish: their role in host defense". Plant Cell 7 (1995): 573588.

34. Thevissen K., et al. "Interactionso antifungal plant defensins with fungal membrane components". Peptides 24 (2003): 1705-1712.

35. Thomma BPHJ., et al. "The complexity of disease signaling in Arabidopsis". Current Opinion in Immunology 13 (2001): 6368.

36. Van der Weerden NL., et al. "The plant defensin NaD1 enters the cytoplasm of Fusarium oxysporum hyphae". Journal of Biochemistry 283 (2008): 14445-14452.

37. Wang YP., et al. "Constitutive expression of pea defensin gene DRR206 confers resistance to blackleg (Leptosphaeriamaculans) disease in transgenic canola (Brassica napus)". Molecular Plant Microbe Interaction 12 (1999): 410-418.

38. Wu J., et al. "A plant defensin gene from Orychophragmus violaceus can improve Brassica napus resistance to Sclerotinia sclerotiorum". African Journal of Biotechnology 8.22 (2009): 6101-6109.

39. Zhang Y., et al. "The genetic and molecular basis of plant resistance to pathogens". Journal of Genetics and Genomics 40 (2013): 23-35.

\section{Assets from publication with us}

- Prompt Acknowledgement after receiving the article

- Thorough Double blinded peer review

- Rapid Publication

- Issue of Publication Certificate

- High visibility of your Published work

Website: www.actascientific.com/

Submit Article: www.actascientific.com/submission.php

Email us: editor@actascientific.com

Contact us: +919182824667

Citation: GDG Chaturani., et al. "Two Novel Defensin Genes from Brassica Juncea and Camelina Sativa Confers Antifungal Activity Against Pathogenic Fungi Alernaria brassicae". Acta Scientific Agriculture 6.2 (2022): 40-49. 